\title{
Highly Specific Memory B Cells Generation after the 2nd Dose of BNT162b2 Vaccine Compensate for the Decline of Serum Antibodies and Absence of Mucosal IgA
}

\author{
Eva Piano Mortari ${ }^{1,+}+\left(\mathbb{D}\right.$, Cristina Russo ${ }^{2,+}$, Maria Rosaria Vinci ${ }^{3,4,+}$, Sara Terreri ${ }^{1}$, Ane Fernandez Salinas ${ }^{1,5}$, \\ Livia Piccioni ${ }^{2}$, Claudia Alteri ${ }^{1,6}$, Luna Colagrossi ${ }^{2}$, Luana Coltella ${ }^{2} \odot$, Stefania Ranno ${ }^{2}$, Giulia Linardos ${ }^{2}$, \\ Marilena Agosta ${ }^{2}$, Christian Albano ${ }^{1}$, Chiara Agrati ${ }^{7}$, Concetta Castilletti ${ }^{7}\left(\mathbb{D}\right.$, Silvia Meschi ${ }^{7}$, \\ Paolo Romania ${ }^{1,5}$, Giuseppe Roscilli ${ }^{8}$, Emiliano Pavoni ${ }^{8}$, Vincenzo Camisa ${ }^{3,4}$, Annapaola Santoro ${ }^{3,4}$, \\ Rita Brugaletta ${ }^{3,4}$, Nicola Magnavita 9,10®), Alessandra Ruggiero ${ }^{11,12}\left(\right.$, Nicola Cotugno ${ }^{11}\left(\right.$, Donato Amodio ${ }^{11}$, \\ Marta Luisa Ciofi Degli Atti ${ }^{13}$, Daniela Giorgio ${ }^{14}$, Nicoletta Russo ${ }^{14}$, Guglielmo Salvatori ${ }^{14}$, Tiziana Corsetti ${ }^{15}$, \\ Franco Locatelli ${ }^{16,17 \mathbb{D}}$, Carlo Federico Perno ${ }^{1,2, \ddagger}$, Salvatore Zaffina ${ }^{3,4, \neq}$ and Rita Carsetti ${ }^{1,2, *, \neq}$
}

check for

updates

Citation: Piano Mortari, E.; Russo,

C.; Vinci, M.R.; Terreri, S.; Fernandez Salinas, A.; Piccioni, L.; Alteri, C.; Colagrossi, L.; Coltella, L.; Ranno, S.; et al. Highly Specific Memory B Cells Generation after the 2nd Dose of BNT162b2 Vaccine Compensate for the Decline of Serum Antibodies and Absence of Mucosal IgA. Cells 2021, 10, 2541. https://doi.org/10.3390/ cells10102541

Academic Editors: Zdenek Hel and Alessandro Poggi

Received: 25 August 2021

Accepted: 22 September 2021

Published: 26 September 2021

Publisher's Note: MDPI stays neutral with regard to jurisdictional claims in published maps and institutional affiliations.

Copyright: (c) 2021 by the authors. Licensee MDPI, Basel, Switzerland. This article is an open access article distributed under the terms and conditions of the Creative Commons Attribution (CC BY) license (https:/ / creativecommons.org/licenses/by/ $4.0 /$ )
1 Diagnostic Immunology Research Unit, Multimodal Medicine Research Area, Bambino Gesù Children's Hospital, IRCCS, Viale di San Paolo,15, 00146 Rome, Italy; eva.pianomortari@gmail.com (E.P.M.); sara.terreri@opbg.net (S.T.); ane.fernandezsalinas@opbg.net (A.F.S.); claudia.alteri@unimi.it (C.A.); christian.albano@opbg.net (C.A.); paolo.romania@uniroma1.it (P.R.); carlofederico.perno@opbg.net (C.F.P.)

2 Microbiology and Diagnostic Immunology Unit, Bambino Gesù Children's Hospital, IRCCS, Piazza Sant'Onofrio, 4, 00165 Rome, Italy; cristina.russo@opbg.net (C.R.); livia.piccioni@opbg.net (L.P.); luna.colagrossi@opbg.net (L.C.); luana.coltella@opbg.net (L.C.); stefania.ranno@opbg.net (S.R.); giulia.linardos@opbg.net (G.L.); marilena.agosta@opbg.net (M.A.)

3 Occupational Medicine/Health Technology Assessment and Safety Research Unit, Clinical-Technological Innovations Research Area, Bambino Gesù Children's Hospital, IRCCS, Viale di San Paolo, 15, 00146 Rome, Italy; mariarosaria.vinci@opbg.net (M.R.V.); vincenzo.camisa@opbg.net (V.C.); annapaola.santoro@opbg.net (A.S.); rita.brugaletta@opbg.net (R.B.); salvatore.zaffina@opbg.net (S.Z.)

4 Health Directorate, Bambino Gesù Children's Hospital, IRCCS, Piazza Sant'Onofrio, 4, 00165 Rome, Italy

5 Department of Molecular Medicine, Sapienza University of Rome, Viale dell'Università, 37, 00185 Rome, Italy

6 Department of Oncology and Hemato-Oncology, University of Milan, Via festa del Perdono, 7, 20122 Milan, Italy

7 National Institute for Infectious Diseases Lazzaro Spallanzani, IRCCS, Via Portuense, 2, 00146 Rome, Italy; chiara.agrati@inmi.it (C.A.); concetta.castilletti@inmi.it (C.C.); silvia.meschi@inmi.it (S.M.)

8 Takis s.r.l., Via di Castel Romano, 100, 00128 Rome, Italy; roscilli@takisbiotech.it (G.R.); pavoni@takisbiotech.it (E.P.)

9 Section of Occupational Medicine and Labor Law, Post-Graduate School of Occupational Health, University Cattolica del Sacro Cuore, Largo Francesco Vito, 1, 00168 Rome, Italy; nicola.magnavita@policlinicogemelli.it

10 Department of Woman, Child \& Public Health, Fondazione Policlinico Universitario A. Gemelli IRCCS, Via della Pineta Sacchetti, 217, 00168 Rome, Italy

11 Academic Department of Pediatrics (DPUO), Research Unit of Clinical Immunology and Vaccinology, Bambino Gesù Children's Hospital, IRCCS, 00165 Rome, Italy; alessandra.ruggiero@opbg.net (A.R.); nicola.cotugno@opbg.net (N.C.); donato.amodio@opbg.net (D.A.)

12 Department of Neuroscience, Biomedicine and Movement Sciences, University of Verona, Via San Francesco, 22, 37129 Verona, Italy

13 Clinical Pathways and Epidemiology Unit, Bambino Gesù Children's Hospital, IRCCS, Piazza Sant'Onofrio, 4, 00165 Rome, Italy; marta.ciofidegliatti@opbg.net

14 Neonatal Intensive Care Unit and Human Milk Bank, Department of Neonatology, Bambino Gesù Children's Hospital, IRCSS, Piazza Sant'Onofrio, 4, 00165 Rome, Italy; daniela.giorgio@opbg.net (D.G.); nicoletta.russo@opbg.net (N.R.); guglielmo.salvatori@opbg.net (G.S.)

15 Hospital Pharmacy Unit, Bambino Gesù Children's Hospital, IRCCS, Piazza Sant'Onofrio, 4, 00165 Rome, Italy; tiziana.corsetti@opbg.net

16 Department of Hematology/Oncology, Bambino Gesù Children's Hospital, IRCCS, Piazza Sant'Onofrio, 4, 00165 Rome, Italy; franco.locatelli@opbg.net

17 Department of Pediatrics, Sapienza, University of Rome, Viale dell'Università, 37, 00185 Rome, Italy

* Correspondence: rita.carsetti@opbg.net

+ Co-first author: These authors contributed equally to this work.

$\ddagger$ Co-last author: These authors contributed equally to this work. 


\begin{abstract}
Specific memory B cells and antibodies are a reliable read-out of vaccine efficacy. We analysed these biomarkers after one and two doses of BNT162b2 vaccine. The second dose significantly increases the level of highly specific memory B cells and antibodies. Two months after the second dose, specific antibody levels decline, but highly specific memory B cells continue to increase, thus predicting a sustained protection from COVID-19. We show that although mucosal IgA is not induced by the vaccination, memory B cells migrate in response to inflammation and secrete $\operatorname{Ig} \mathrm{A}$ at mucosal sites. We show that the first vaccine dose may lead to an insufficient number of highly specific memory B cells and low concentration of serum antibodies, thus leaving vaccinees without the immune robustness needed to ensure viral elimination and herd immunity. We also clarify that the reduction of serum antibodies does not diminish the force and duration of the immune protection induced by vaccination. The vaccine does not induce sterilizing immunity. Infection after vaccination may be caused by the lack of local preventive immunity because of the absence of mucosal IgA.
\end{abstract}

Keywords: memory B cells; BNT162b2; IgA; vaccine; SARS-CoV-2; immunity

\title{
1. Introduction
}

The SARS-CoV-2 pandemic continues to spread and cause COVID-19 and death. Hospitals are under pressure, and the economic damage is unprecedented. Vaccination is the safest and most effective tool to achieve a protective response in most individuals. Effective vaccines against SARS-CoV-2 have been rapidly developed and are being administered with the aim of preventing COVID-19, stopping viral circulation and terminating the pandemic. Phase III trials have demonstrated the high efficacy of two-dose regimens [1-3]. The available data have shown that all available vaccines are protective against hospitalization and severe illness after completing the appropriate immunisation schedule. According to data provided by Mathieu et al. (2021) [4], in mid-July 2021, about $26 \%$ of the world population had received at least one dose of a COVID-19 vaccine. Unvaccinated or not fully vaccinated individuals with an immune system unable to clear the infection may favour the emergence of viral variants [5]. From the epidemiological point of view, it has been predicted that a one-dose regimen will foster antigenic evolution and weaken the protective potentials of the available vaccines and of those still in development, all based on the sequence of a virus that may have changed under the selective pressure of an immune response unable to completely clear the virus [6]. Several variants of concern (VoC) have been identified and have spread throughout the world. New variants are continuously generated [7].

Another still open question is the duration of protective immunity after vaccination. Most attention has been concentrated on the level of specific antibodies, which increase in response to the vaccine, but decline after a few months. The reduction of specific antibody levels generates considerable anxiety in the population because it is perceived as incipient loss of protection.

Antibody decline is, however, a normal feature of the response to every vaccination [8]. Our most important protection from reinfection is represented by the synergistic action of memory B cells (MBCs) and memory plasma cells. Specific antibodies present in the serum are continuously secreted by memory plasma cells and face the pathogen at its first entry [9]. At the same time, MBCs migrate in response to chemokines to the inflamed tissues and reinforce protection by locally secreting antibodies. If the antibodies produced by MBCs and plasma cells have a high affinity for the pathogen, this is immediately eliminated [8]. T cells play a fundamental role by helping $B$ cells to produce high-affinity antibodies and MBCs $[10,11]$ and, most importantly, by eliminating virus-infected cells [12].

From the point of view of B-cell immunology, in order to support public health decisions and measures, the most important issues to be addressed are: 1 . the affinity of MBCs and plasma cells generated after the 1st and 2nd vaccine dose; 2 . the longevity 
of vaccine-induced immune memory; 3 . the ability of the vaccine to induce sterilizing immunity, thus preventing not only severe COVID-19, but also infection.

We addressed these three points in a study on the B-cell response to the first and second dose of BNT162b2 vaccine and a further evaluation of the established protection three months after the 1 st dose.

We report the effects of the two-doses regimen of BNT162b2 vaccine in a population of Health Care Workers (HCWs) of the Bambino Gesù Children Hospital IRCCS.

In $108 \mathrm{HCWs}$, we measured specific MBCs by ELISpot, flow cytometry and serum antibodies at different time points, before and after first and second dose and three months later. We found that one single dose results in immune memory lacking the precision indispensable to immediately clear the virus that is the basis for vaccine effectiveness. The second dose increases the frequency of vaccine responders and specific IgG levels and generates highly specific MBCs. Three months later, serum antibodies significantly decline, but, in contrast, MBCs increase in frequency and affinity. Most importantly, the vaccine triggers a serological IgA response but does not generate mucosal IgA. The lack of specific $\operatorname{IgA}$ strategically located at the virus site of entrance explains why the vaccine does not induce sterilizing immunity.

\section{Materials and Methods}

\subsection{Ethical Approval}

Ethical approval was obtained from the Ethics Committee at OPBG, Bambino Gesù Children Hospital. According to the guidelines on Italian observational studies as established by the Italian legislation about the obligatory occupational surveillance and privacy management, the confidentiality of HCWs was safeguarded, and informed consent was obtained from all the participants. The study was performed in accordance with the Good Clinical Practice guidelines, the International Conference on Harmonization guidelines, and the most recent version of the Declaration of Helsinki.

\subsection{Human Subjects}

We included in our study $108 \mathrm{HCWs}$ (Table 1) who had never experienced SARS-CoV2 infection before, as demonstrated by molecular (Allplex2019-ncov, Seegene, Seoul, South Korea) and antibody assays (Elecsys ${ }^{\circledR}$ Anti-N, Roche, Basel, Switzerland).

Table 1. Characteristics of the study population.

\begin{tabular}{ccccc}
\hline \multicolumn{2}{c}{ Study Group } & Memory B Cells by ELISpot & Memory B Cells by FACS & Surrogate Neutralization \\
\hline Number of HCW & 108 & 74 & 34 & 70 \\
\hline Age (mean \pm SD) & $46.95 \pm 11.35$ & $47.04 \pm 10.93$ & $45.7 \pm 11.96$ & $48.11 \pm 11.45$ \\
\hline Sex (M/F) & $31 / 77$ & $17 / 57$ & $14 / 20$ & $17 / 53$ \\
\hline
\end{tabular}

We analysed peripheral blood from 108 HCWs of the Bambino Gesù Children Hospital (Tables 1 and 2). We included in the study all HCWs that were vaccinated by two injections of BNT162b2, $30 \mu \mathrm{g}$, 21 days apart as prescribed by the immunization schedule. BNT162b2 vaccine encodes a P2 mutant spike protein (PS2) and is formulated as an RNA-lipid nanoparticle (LNP) of nucleoside-modified mRNA (modRNA). A two-dose regimen of BNT162b2 conferred 95\% protection against COVID-19 (95\% CI, 90.3 to 97.6) [1].

Blood and serum samples were collected at defined intervals after vaccination. Samples were available before first dose (T0) and at 7 (T7), 21 (T21), 28 (T28) and 90 (T90) days after the first dose (Figure 1). 
Table 2. Study cohort used in the different assays.

\begin{tabular}{lcc}
\hline \multirow{3}{*}{ Number of patients } & Assay & \multicolumn{1}{c}{$\begin{array}{c}\text { Total Patients Followed Over } \\
\text { Time }\end{array}$} \\
\cline { 2 - 3 } & ELISpot & 74 \\
\cline { 2 - 3 } & FACs & 34 \\
\cline { 2 - 3 } & Surrogate Neutralization & 70 \\
\cline { 2 - 3 } & ELISA & 108 \\
\hline
\end{tabular}

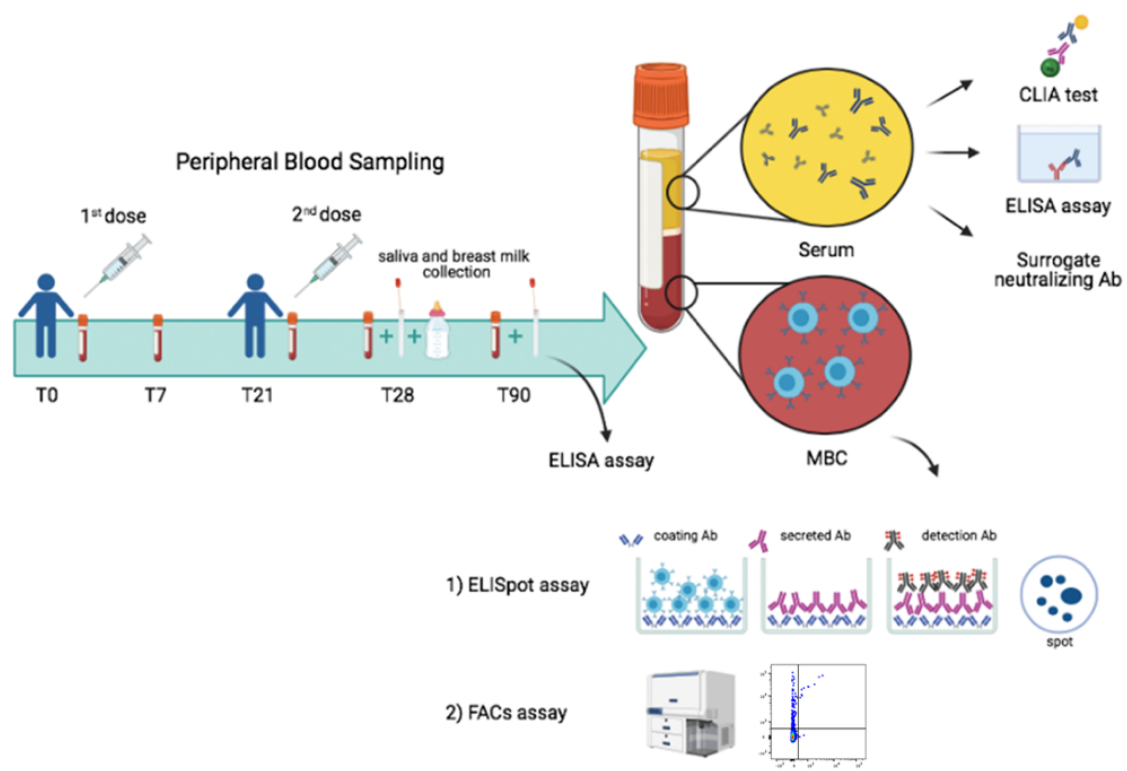

Figure 1. Study design. Design of our vaccine study indicating timeline and experimental plan.

At the time of blood sampling, none of the subjects had an acute infection or were taking any medication known to alter immune function (such as steroids or statins).

Specific antibodies were measured in the serum, saliva and breast milk (Figure 1). MBCs were identified by two different methods. By ELISpot, after polyclonal stimulation with $C p G$, we detected MBCs based on their ability to secrete antibodies that bind the vaccine target spike protein on coated plates. We also identified MBCs ex vivo by flow cytometry, thanks to the availability of biotin-labelled recombinant spike protein coupled to a very high brightness fluorescence dye (PE) and the same biotin-labelled spike coupled to a moderate brightness fluorescent dye (BUV395) [13]. We were able to distinguish MBCs with low (PE single positive, $\mathrm{S}+$ ) or high affinity (PE-BUV395 double positive, $\mathrm{S}++$ ) for recombinant spike.

Given the limited amount of blood available for the study, we randomly selected 74 HCWs samples for the ELISpot and 34 for FACs (Table 2). Serology was performed in all samples and surrogate neutralization in 70 samples and only at T21, T28 and T90 (Table 2).

Breast milk was obtained from $23 \mathrm{HCWs}$, of which 16 were vaccinated during lactation (Table 3).

Table 3. Characteristics of enrolled mothers.

\begin{tabular}{cc}
\hline & Study Group \\
\hline Mothers' age (mean $\pm \mathrm{SD})$ & $33.36 \pm 4.17$ \\
\hline Mothers' breast milk & 7 not vaccinated \\
& 16 vaccinated \\
\hline
\end{tabular}


Saliva samples were collected by aspirating with a $1 \mathrm{~mL}$ syringe from the oral cavity at least 30 min after eating, drinking, smoking or chewing gum. Saliva was immediately transferred to an Eppendorf tube and frozen at $-20{ }^{\circ} \mathrm{C}$ until use.

\subsection{Cell Isolation and Cryopreservation}

Heparinized PBMCs were isolated by Ficoll Paque ${ }^{\mathrm{TM}}$ Plus 206 (Amersham PharmaciaBiotech, Amersham, UK) density-gradient centrifugation and immediately frozen and stored in liquid nitrogen until use. The freezing medium contained $90 \%$ Foetal Bovine Serum (FBS) and 10\% DMSO.

\subsection{Polyclonal Memory B-Cell Stimulation}

As we published before [14], in order to induce antibody production from MBCs, we polyclonally stimulated PBMCs with CpG. Briefly, PBMCs were cultured in complete medium at a concentration of $1 \times 10^{6}$ cells $/ \mathrm{mL}$. Complete medium was prepared as follows: RPMI-1640 (Euroclone, Milan, Italy), 10\% heat inactivated HyClone FBS (Hyclone Laboratories, Logan, UT, USA), 1mM L-Glutamine (GIBCO BRL, New York, NY, USA); 1 $\times$ Penicillin/Streptomycin $100 \times$ (Euroclone, Milan, Italy), 1\% sodium pyruvate (GIBCO BRL, New York, NY, USA). Cells were stimulated for 5 days with $0.35 \mu \mathrm{M}$ TLR9 agonist CpG-B ODN2006 (Hycult Biotech, Uden, The Netherland) plus 20 ng/mL rhIL-21 (Milteny, Bergisch Gladbach, Germany) and 20 ng/mL rhIL-4 (Milteny, Bergisch Gladbach, Germany).

\subsection{B Cell ELISpot}

The B cell (enzyme-linked immunospot) ELISpot assay was performed with 5 days stimulated-PBMCs for the detection of MBCs.

96-well MultiScreen filter plates (Millipore, Burlington, NJ, USA) were pre-wetted with $35 \%$ ethanol for $\leq 1 \mathrm{~min}$ and washed with $\mathrm{ddH}_{2} \mathrm{O}(5 \times 200 \mu \mathrm{L} /$ well $)$. Wells were coated with $50 \mu \mathrm{L}$ PBS containing either isotype-specific AffiniPure $\mathrm{F}\left(\mathrm{ab}^{\prime}\right)_{2}$ fragment goat anti-human antibody (Jackson ImmunoResearch Laboratories, Cambridge, UK) (anti-IgM final concentration $2.5 \mu \mathrm{g} / \mathrm{mL}$, anti-IgG $15 \mu \mathrm{g} / \mathrm{mL}$, anti-IgA $10 \mu \mathrm{g} / \mathrm{mL}$ ); or recombinant spike (R\&D Systems) at a final concentration of $1 \mu \mathrm{g} / \mathrm{mL}$ to detect specific responses. The plates were then incubated overnight $(\mathrm{ON})$ at $4{ }^{\circ} \mathrm{C}$. After washing with PBS $(5 \times 200$ $\mu \mathrm{L} /$ well $)$ and blocking with PBS $+1 \%$ BSA $+5 \%$ Sucrose $(5 \times 200 \mu \mathrm{L} /$ well $)$ for $2 \mathrm{~h}$ at RT, plates were filled with sterile RPMI $+10 \%$ FBS $(100 \mu \mathrm{L} /$ well $)$ for $1 \mathrm{~h}$ at $37^{\circ} \mathrm{C}$.

After 5 days of stimulation (as previously described), cells were harvested and washed once with RPMI before counting. Different concentrations of PBMCs were used depending on the following conditions: total IgM, IgG and IgA: $1.5 \times 10^{4}$ PBMCs/well; specific IgM: $5 \times 10^{4}$ PBMCs/well; specific IgG and IgA: 1-5 × 105 PBMCs/well. Four steps of two-fold serial titrations were performed for each condition. Plates were incubated $\mathrm{ON}$ at $37^{\circ} \mathrm{C}$. Cells were discarded, and after washing with PBS $+0.05 \%$ Tween $(5 \times 200 \mu \mathrm{L} /$ well $)$, plates were incubated for $1 \mathrm{~h}$ at $37^{\circ} \mathrm{C}$ with peroxidase-conjugated goat anti-human IgM, IgG or IgA antibodies (Jackson ImmunoResearch Laboratories, Cambridge, UK) diluted in PBS $+0.05 \%$ Tween $+1 \%$ BSA. The plates were washed with PBS $+0.05 \%$ Tween $(5 \times 200$ $\mu \mathrm{L} /$ well) before TMB substrate (Mabtech, Stockholm, Sweden) addition and incubated for $4 \mathrm{~min}$ at RT in the dark. After stopping the reaction by rinsing with $\mathrm{ddH}_{2} \mathrm{O}(5 \times 200$ $\mu \mathrm{L} /$ well), plates were left to dry $\mathrm{ON}$ at $4{ }^{\circ} \mathrm{C}$.

All plates were analysed using an automated AELVIS ELISpot reader (A.EL.VIS GmbH, Germany). Total IgM, IgG and IgA were used as positive controls for each subject at each time point. Samples were excluded if they generated low total Ig responses. For statistical analysis, the frequency of antigen-specific MBCs was reported as percentage $(\%)$ of total MBCs producing antibodies of $\operatorname{IgM}, \operatorname{IgG}$ or $\operatorname{IgA}$ isotype in our culture conditions [15]. 


\subsection{ELISA for Specific IgA and IgM Detection}

A semi-quantitative in vitro determination of human IgA antibodies against the S1 subunit of SARS-CoV-2 was evaluated on serum, saliva and breast milk samples by using the Anti-SARS-CoV-2 ELISA (Euroimmun, Lübeck, Germany), according to the manufacturer's instructions. Values were then normalized for comparison with a calibrator. Results were evaluated by calculating the ratio between the extinction of samples and the extinction of the calibrator. Results are reported as ratio between OD sample and OD calibrator. The ratio interpretation was as follows: $<0.8=$ negative, $\geq 0.8$ to $<1.1=$ borderline, $\geq 1.1=$ positive [16].

To detect IgM anti RBD, we developed an in-house ELISA. 96-well plates (Corning, New York, NY, USA) were coated for $1 \mathrm{~h}$ at $37^{\circ} \mathrm{C}$ with $1 \mu \mathrm{g} / \mathrm{mL}$ of purified SARS-CoV-2 RBD protein (Sino Biological, Beijing, China). After washing with PBS $1 \times / 0.05 \%$ Tween and blocking with PBS $1 \times / 1 \%$ BSA, plates were incubated for $1 \mathrm{~h}$ at $37^{\circ} \mathrm{C}$ with diluted sera (1:100). After washing again, plates were incubated for $1 \mathrm{~h}$ at $37^{\circ} \mathrm{C}$ with peroxidaseconjugated goat anti-human IgM antibody (Jackson ImmunoResearch Laboratories, Cambridge, UK). The assay was developed with o-phenylen-diamine tablets (Sigma-Aldrich, St. Louis, MO, USA) as a chromogen substrate. Absorbance at $450 \mathrm{~nm}$ was measured, and IgM concentrations were calculated by interpolation from the standard curve based on serial dilutions of monoclonal human IgM antibody against SARS-CoV-2 Spike-RBD (Invivogen, San Diego, CA, USA) [16].

\subsection{Quantitative Determination of Anti-N, Anti-S, Trimeric Spike and RBD Antibodies}

Serum samples were tested by two different methods and analytical platforms. Qualitative detection of antibodies direct against the nucleocapsid $(\mathrm{N})$ protein and semiquantitative detection of total antibodies ( $\operatorname{IgA}, \operatorname{IgM}, \operatorname{IgG}$ ) directed against the RBD of the virus spike (S) protein of SARS-CoV-2 were tested by an electro-chemiluminescence sandwich immunoassay (ECLIA), using Elecsys-anti SARS-CoV-2 and Elecsys-anti SARSCoV-2 S (Roche Diagnostics) tests on a Cobas e801 analyser following the manufacturer's instructions. Results for anti-N antibodies are expressed as "present" or "absent" on the basis of a cut-off index (COI) of COI $\geq 1.0$ and COI $<1.0$, respectively. Detection and quantification were automatically calculated for each sample in $\mathrm{U} / \mathrm{mL}$, equivalent to the Binding Arbitrary Unit (BAU) $/ \mathrm{mL}$ of the first WHO International Standard for anti-SARS-CoV-2 immunoglobulins. Titre was interpreted as absent when $<0.8 \mathrm{U} / \mathrm{mL}(<0.8 \mathrm{BAU} / \mathrm{mL})$ and as present when $\geq 0.80 \mathrm{U} / \mathrm{mL}(\geq 0.8 \mathrm{BAU} / \mathrm{mL})$. When the antibodies' titre was higher than $250 \mathrm{U} / \mathrm{mL}$ (250 BAU / mL), the instrument automatically executed a 20-fold dilution, ranging the upper limit of quantification to $5000 \mathrm{U} / \mathrm{mL}(5000 \mathrm{BAU} / \mathrm{mL}$ ). The quantitative determination of anti-trimeric spike protein specific IgG antibodies to SARS-CoV-2 was run on LiaisonXL platform by a new generation of chemiluminescence immunoassay (CLIA) TrimericS IgG assay (DiaSorin, Salluggia, Italy) with a quantification range between 4.81 $\mathrm{BAU} / \mathrm{mL}$ and $2080 \mathrm{BAU} / \mathrm{mL}$ (dilution factor 1:20).

The SARS-CoV-2 IgG II kit, a chemiluminescence microparticle antibody assay detecting antibodies against the RBD of SARS-CoV-2 (CMIA, IgG antibody concentrations expressed as arbitrary units, $\mathrm{AU} / \mathrm{mL} \geq 50$ are considered positive, ARCHITECT $^{\circledR}{ }^{\mathrm{i}}$ 2000sr Abbott Diagnostics), was used according to the manufacturer's protocols.

\subsection{Detection of Antigen-Specific Memory B Cells}

To detect SARS-CoV-2 specific B cells, biotinylated protein antigens were individually multimerized with fluorescently labelled streptavidin at $4{ }^{\circ} \mathrm{C}$ for one hour [17]. Recombinant biotinylated SARS-CoV-2 spike proteins (S1+S2; aa16-1211) were purchased from R\&D systems. RBD was generated in-house and biotinylation was performed using EZ-Link ${ }^{\mathrm{TM}}$ Sulfo-NHS-LC-Biotin reaction kit (ThermoScientific, Waltham, MA, USA) following the manufacturer's standard protocol and dialyzed overnight against PBS. Biotinylated spike was mixed with streptavidin BUV395 (BD) and streptavidin PE (BD) at a 25:1 ratio and 20:1 ratio, respectively. Biotinylated RBD was mixed with streptavidin FITC (BD) at a 2.5:1 ratio. 
Streptavidin PE-Cy7 (BD) was used as a decoy probe to gate out SARS-CoV-2 non-specific streptavidin-binding B cells. The antigen probes prepared individually as above were then mixed in Brilliant Buffer (BD). A total of $\sim 5 \times 10^{6}$ previously frozen PBMC samples were prepared and stained with $85 \mu \mathrm{L}$ antigen probe cocktail containing $100 \mathrm{ng}$ spike per probe (total $200 \mathrm{ng}$ ), $27.5 \mathrm{ng}$ RBD and $20 \mathrm{ng}$ SA-PE-Cy7 at $4{ }^{\circ} \mathrm{C}$ for $30 \mathrm{~min}$ to ensure maximal staining quality before surface staining with antibodies; as listed in Table S1, this was performed in Brilliant Buffer at $4{ }^{\circ} \mathrm{C}$ for $30 \mathrm{~min}$. SARS-CoV-2-specific MBCs were defined as CD19+CD24+CD27+CD38-Spike+ (S+) or CD19+CD24+CD27+CD38-Spike++ (S++).

Stained PBMC samples were acquired on FACS LSRFortessa (BD). At least $2 \times 10^{6}$ cells were acquired and analysed using FlowJo10.7.1 (BD). Phenotype analysis of antigen-specific $B$ cells was performed only in subjects with at least 10 cells detected in the respective antigen-specific memory $B$ cell gate.

\subsection{SARS-CoV-2 Variant Determination}

SARS-CoV-2 variant determination was performed by single-nucleotide-polymorphism (SNP) detection approach. In particular, the CE-approved Real-Time PCR COVID-19 Variant Catcher (Clonit Srl, Milan, Italy, CE IVD), able to detect the spike 69-70del, E484K and N501Y, was used to discriminate between the alpha and the beta/gamma strains. Briefly, viral RNA was extracted, retrotranscribed and amplified according to manufacturer's protocol. Real-time PCR was performed on CFX96 (Bio Rad Laboratories, Hercules, CA, USA). In order to further discriminate between beta and gamma, the presence of SNP H655Y and A701V were further investigated by means of homemade sanger sequencing protocol. In brief, the spike region encompassing amino acids 605-900 was retrotranscribed, amplified and sequenced by using two different sequence-specific primers (FWD: 5'TACTTCTAACCAGGTTGCT3'; REV: 5'CTATAAGCCATTTGCATAGCA3'). Sequences were obtained thanks to the automated sequencer ABI-3130 (Applied Biosystems, Waltham, MA, USA). Negative and positive controls were included in each run to monitor carry-over contamination and PCR efficiency.

\subsection{Statistical Analysis}

Values were compared by the non-parametric Kruskal-Wallis test and, if not significant, the Wilcoxon matched pair signed-rank test was used for the comparison between time points of each subject. Pairwise comparisons were evaluated by the Mann-Whitney $\mathrm{U}$ test nonparametric test. The Chi-square test with $2 \times 2$ contingency tables was used to compare pie charts. $p$-values of $<0.05$ were considered statistically significant. Statistical analyses were performed with GraphPad Prism 8.0 (GraphPad Software).

The relationship between variables was studied using a correlation test based on the Pearson's product moment correlation coefficient $\operatorname{cor}(x, y)$ and follows a t distribution with length (x)-2 degrees of freedom if the samples follow independent normal distributions. If there are at least 4 complete pairs of observation, an asymptotic confidence interval is given based on Fisher's Z transform (https: / / stat.ethz.ch/R-manual/R-devel/library/ stats/html/cor.test.html, accessed on 17 May 2021).

\section{Results}

\subsection{Serum Antibodies}

Serum antibodies are the most commonly used surrogate biomarkers of vaccine efficacy. We measured specific serum antibodies using different methods.

Anti-RBD antibodies of IgM isotype were measured by ELISA. We found that antiRBD IgM was present at low levels at T0 and T7, reflecting the presence of natural $[10,18]$ or cross-reactive antibodies $[19,20]$. The increase at T21 and T28 is the effect of the vaccination. At T90, anti-RBD IgM slightly declined (Figure 2A).

Anti-RBD antibodies of all isotypes, evaluated by Elecsys ${ }^{\circledR}$ Anti-SARS-CoV-2-S (Roche, cut-off: $0.8 \mathrm{BAU} / \mathrm{mL}$ ), were absent at T0 and T7 but already increased at T21. Their level, however, was much higher at T28 and decreased at T90 (Figure 2B). The geometric 
mean at T21 was 29.13 BAU/mL and became $1736 \mathrm{BAU} / \mathrm{mL}$ at T28, demonstrating the importance of the booster dose. At T90, the geometric mean decreased to $819.8 \mathrm{BAU} / \mathrm{mL}$. Similar results were obtained when we measured IgG against trimeric spike (DiaSorin, cut-off: 13 BAU/mL). The geometric mean values increased from $205.9 \mathrm{BAU} / \mathrm{mL}$ at T21 to $1660 \mathrm{BAU} / \mathrm{mL}$ at T28 and then decreased to $991.3 \mathrm{BAU} / \mathrm{mL}$ at T90 (Figure 2C).
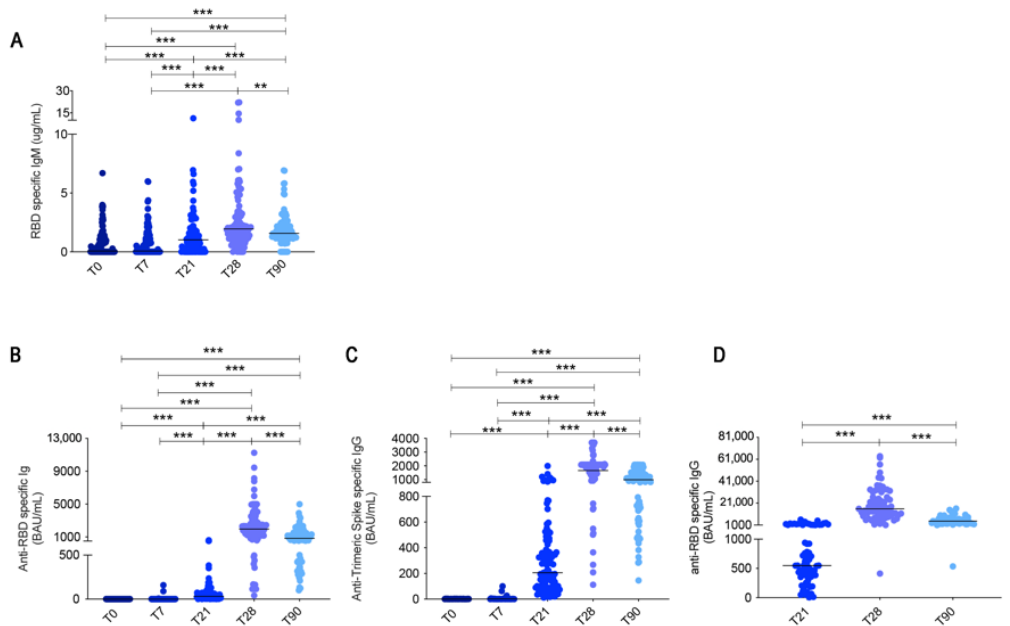

E
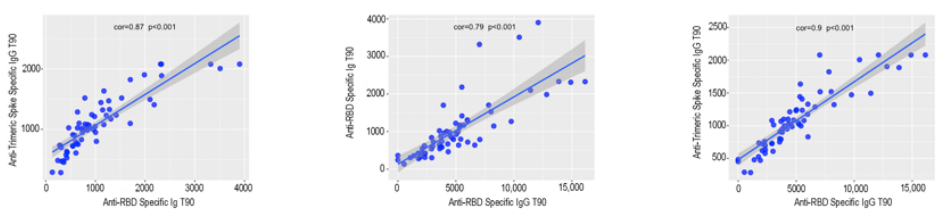

Figure 2. SARS-CoV-2 antibody responses. (A) Anti-RBD IgM was measured by an in-house ELISA. (B) Anti-RBD and (C) anti-trimeric $S$ titres were assessed by Elecsys ${ }^{\circledR}$ Anti-SARS-CoV-2 S assay (Roche, cut-off $0.8 \mathrm{U} / \mathrm{mL}$ ) and Liaison SARS-CoV-2 trimeric S IgG Assay (Diasorin, cut-off $13 \mathrm{AU} / \mathrm{mL}$ ) before vaccine administration (T0), at 7 (T7), 21 (T21) 28 (T28) and 90 (T90) days after the first dose $(n=108)$. (D) Surrogate neutralization IgG titres at T21, T28 and T90 $(n=70)$. (E) Scatter plots with a regression line of IgG anti-trimeric S vs. Ig anti-RBD; Ig anti-RBD vs. IgG anti-RBD and IgG Anti-trimeric S vs. IgG anti-RBD. On top of the graph, we reported the Pearson's correlation coefficient and the p-value from the correlation test. Medians are plotted and statistical significance was determined using Wilcoxon matched-pairs signed-rank test. ${ }^{* *} p<0.01$, ${ }^{* * *} p<0.001$.

Thus, specific antibodies are already produced after the first vaccine dose. However, anti-RBD antibodies increased 60 times between T21 and T28, and anti-trimeric spike IgG raised eight times after the second dose. Three months after the first dose, anti-RBD antibodies and anti-trimeric spike IgG were reduced by half (Figure 2B,C).

The anti-viral activity of IgG after the first and second dose and at T90 was measured by using, as surrogate virus neutralization assay, a specific chemiluminescence microparticle antibody assay (CMIA) on Abbott ARCHITECT ${ }^{\circledR}$ i2000sr [21,22] that detects IgG antibodies specific for RBD. The geometric mean at day 21 was $559.3 \mathrm{BAU} / \mathrm{mL}$ and increased 25 times at T28 (geometric mean $14129 \mathrm{BAU} / \mathrm{mL}$ ), thus confirming the importance of re-stimulation to obtain a significant amplification of the protective response (Figure 2D). Surrogate neutralization activity was reduced by 3.2-fold at T90 (geometric mean 4403; Figure 2D).

The reduction of antibody levels three months after vaccination reflects the normal kinetics of the serological immune response [23].

There was no correlation between antibody levels measured by the different tests at early times after vaccination, whereas the correlation became significant at T90 (Figure 2E). As the commercial test used here measure slightly different responses (all isotypes against 
RBD, IgG specific for either Trimeric Spike or RBD), the correlation at T90 may reflect the loss of short-lived plasma cells released in the early stages of the immune reaction and the progressive selection of useful IgG antibodies against trimeric spike and RBD.

\subsection{Memory B Cell Detected by ELISpot}

ELISpot to detect B cells able to secrete spike-specific IgM, IgG and IgA antibodies was performed in $74 \mathrm{HCWs}$. We found that a high frequency of IgM MBCs was able to bind recombinant spike protein already at $\mathrm{T} 0$, and their number slightly increased 7 days after the first dose (T7) (Figure 3A). This is an expected result because our pool of IgM MBCs includes a wide range of cross-reactive specificities from which high-affinity MBCs will be shaped and selected by the germinal centre (GC) reaction [11].

A
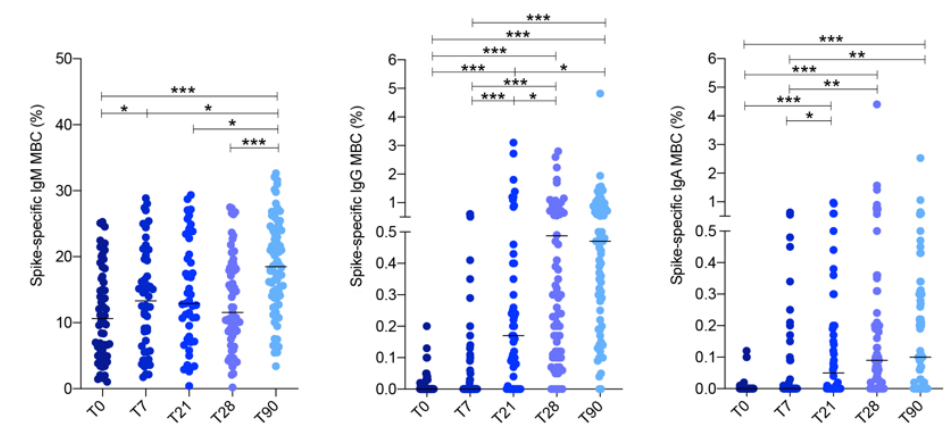

B

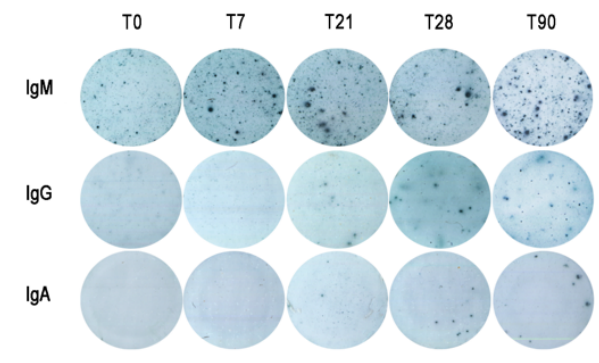

Figure 3. Antigen-specific memory B cell responses after mRNA BNT162b2 vaccination in HCW. (A) Spike-specific MBCs were detected by ELISpot following PBMC stimulation with CpG, IL-21 and IL-4 for 5 days. For each isotype, results are shown as percentage (\%) of MBCs specific for spike over time (T0, T7, T21, T28 and T90). (B) Representative images of spike-specific MBCs detected by ELISpot. Medians are plotted and statistical significance was determined using Wilcoxon matched-pairs signed-rank test. ${ }^{*} p<0.05,{ }^{* *} p<0.01,{ }^{* * *} p<0.001$.

In the GC, the mechanisms of somatic mutation and antigen-driven affinity-maturation improve the specificity of the antibodies, and class-switching generates MBCs and plasma cells able to secrete switched antibodies that have the function of terminating infections and clearing the pathogen.

High specificity and affinity are the most important characteristics of protective MBCs, generated by the adaptive immune system in response to infection or vaccination.

In agreement with the timing of the GC reaction, IgG MBCs specific for spike significantly increased at $\mathrm{T} 21$ after the first dose and significantly more at $\mathrm{T} 28$, seven days after the second dose (Figure 3A). Importantly, we found that $20.8 \%$ of the HCWs did not have IgG-producing MBCs cells in the peripheral blood at T21, following the first dose. Seven days after the second dose (T28), however, IgG-producing MBCs became detectable in the great majority of the HCWs (92.4\%) (Figure 3A,B), again demonstrating the importance of the booster administration.

IgA-secreting MBCs were less frequently detected in the peripheral blood of vaccinated individuals. We found that $58.4 \%$ of the HCWs produced IgA MBCs 21 days after the 
first dose; seven days after the second dose (T28), IgA-producing MBCs were found in the peripheral blood of $71.3 \%$ of the individuals (Figure $3 \mathrm{~A}, \mathrm{~B}$ ).

An important finding of our study is that, in contrast to the decline of specific antibody levels in the serum, MBCs of IgM, IgG and IgA isotypes remained stable three months after the first dose (Figure 3A,B).

Overall, our results show that at all time points of analysis, about $10 \%$ (median) of $\operatorname{IgM}$ MBCs were able to secrete antibodies binding trimeric spike in ELISpot. Our data confirms the role of IgM MBCs as first-line defence [10] and substrate [11] for the generation of high-affinity switched MBCs. The adaptive immune response triggered by the vaccine needs time and is strongly selective [24].

For this reason, IgG and IgA MBCs specific for trimeric spike significantly increase only at T21 and T28 and are rare. In total, $0.17 \%$ of the IgG MBCs are specific for trimeric spike at T21 and $0.48 \%$ at T28. For IgA MBCs, the corresponding medians are $0.05 \%$ at T21 and $0.09 \%$ at T28 (Figure 3A). At T90, there was no further change of IgG and IgA MBCs. IgM MBCs instead significantly increased, suggesting that the MBC pool maintains and expands the newly acquired specificity in its adaptable repertoire, represented by $\operatorname{IgM}$ MBCs [11].

\subsection{Spike-Specific Memory B Cells Detected by Flow Cytometry}

MBCs can be detected in the peripheral blood of convalescent patients and immunized individuals using fluorescent recombinant spike and RBD [17].

We chose to visualize biotin-labelled recombinant spike using two different streptavidinfluorochromes, one with a very high brightness index (streptavidin-PE) and the other with a moderate one (streptavidin-BUV395). Our intention was to visualize low-affinity MBCs detectable with streptavidin-PE (S+) and high-affinity MBCs that would appear as double positive (PE-BUV395; S++). Indeed, in individuals convalescent from COVID-19 infection, most MBCs have a high-affinity and are double positive for PE and BUV395 recombinant spike (S++) (Figure S1).

Among spike-positive MBCs (S+ plus $\mathrm{S}++$ ), we also detected RBD-specific cells with streptavidin-FITC bound to biotin-RBD.

After excluding aspecific binder B cells with streptavidin-PE-Cy7, we gated CD19+ CD24+CD27+CD38- MBCs (Figure 4A) and calculated the frequency of S+ and S++ MBCs and of RDB+ MBCs. We found that low-affinity MBCs (S+) were already detectable at T0 (Figure 4B,C) and were mostly of IgM isotype (Figure 4D), as expected, because of the structure and function of the large repertoire from which we shaped and selected our specific MBCs and in agreement with our ELISpot results (Figure 3A,B). S+ MBCs slightly increased at T7 and more significantly at T21 and T28 (Figure 4E), remaining mostly of IgM isotype (Figure 4F).

S++ MBCs are absent in HCWs who never experienced SARS-CoV-2 infection before, but their development is induced by the vaccine (Figure $4 \mathrm{~B}, \mathrm{C}$ ). Double positive spikespecific MBCs ( +++ ) significantly increased at T21 and even more at T28, seven days after the second dose (Figure 4B,C). Most of them expressed switched isotypes (IgM-) (Figure 4D).

At T90, we found that S+ MBCs significantly declined, whereas the frequency of S++ MBCs increased four-fold (Figure 4B,C). These results reflect the ongoing maturation of the GC response leading to the progressive selection of MBCs that optimally bind the antigen and are mostly of switched isotypes. At T90, the majority of S+ MBCs were still of IgM isotype (Figure $4 \mathrm{D})$.

Among all MBCs able to bind the recombinant spike ( + plus $S++)$, we identified $\mathrm{RBD}+$ cells that represent a minority of the MBCs generated by vaccination. RBD+ MBCs significantly increased at T21 and even more at T28. Their frequency remained stable at T90 (Figure 4E,F). RBD+ MBCs were almost only found in the S++ MBC pool (Figure 4G), indicating the need of the GC mutation and selection mechanisms to generate this new specificity. 
A

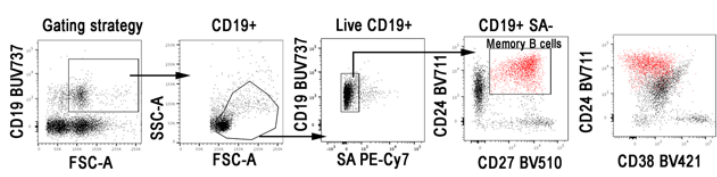

B

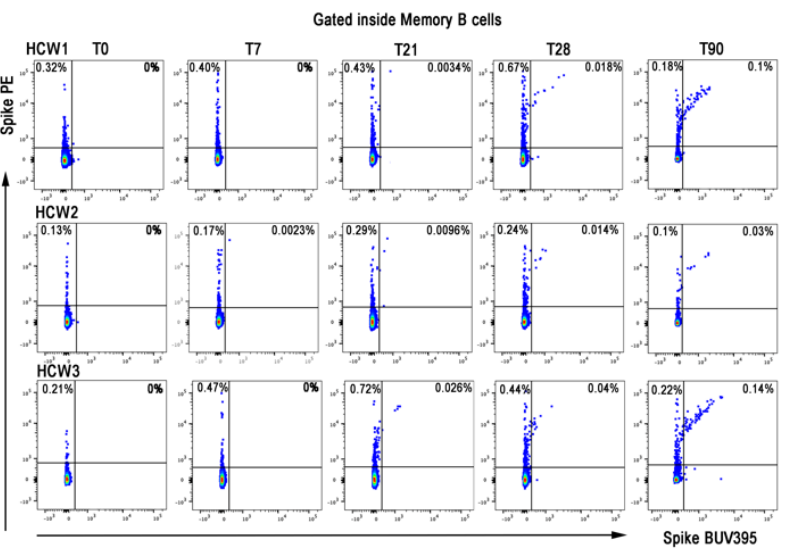

D

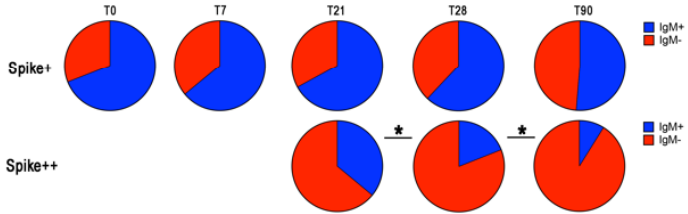

E

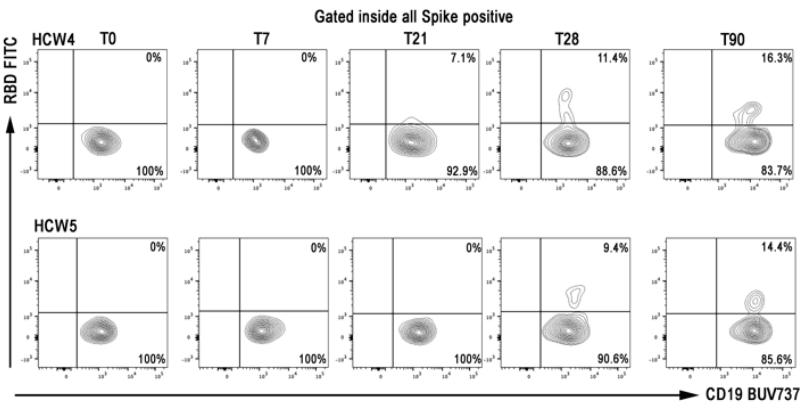

C
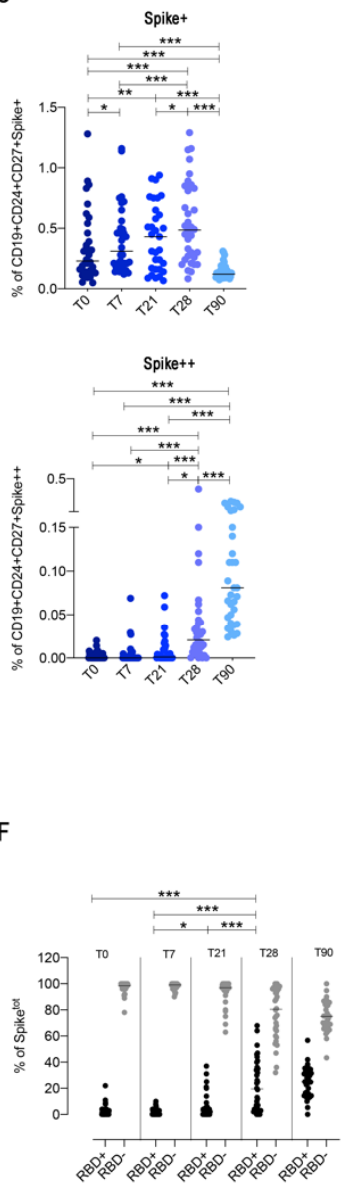

G

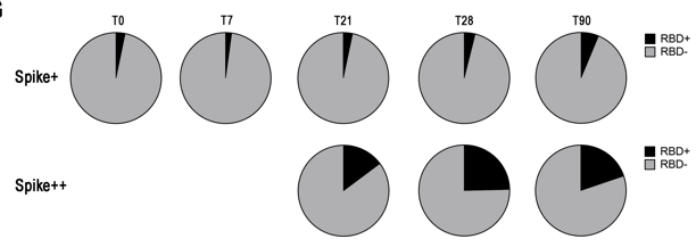

Figure 4. Spike-specific memory B cells. (A) Gating strategy to identify S+ and S++ MBCs. MBCs were identified as CD19+PECy7-CD24+CD27+CD38-. S+ are MBCs cells that are Spike-PE+ but Spike-BUV395-. S++ are instead Spike-PE+ and Spike-BUV395+ (B) Flow cytometry plots in three representative HCWs showing staining patterns of SARS-CoV-2 antigen probes on MBCs during the follow-up. (C) Scatter plots depict the percentage of S+ and S++ inside MBCs $(n=34)$. (D) Pie charts represent the percentage of IgM+ and IgM- S+ and S++ MBCs. (E) Identification of RBD+ cells inside total spike-positive (S+ plus S++) MBCs in two representative HCWs. (F) Scatter plot shows the percentage of RBD+ and RBD- spike-specific MBCs at the different time points of the analysis $(n=34)$. (G) Pie charts show the average percentages of RBD+ and RBD- among MBCs specific for recombinant spike (S+ plus $\mathrm{S}++$ ). Medians are plotted and statistical significance was determined using Wilcoxon matched-pairs signed-rank test. ${ }^{*} p<0.05,{ }^{* *} p<0.01,{ }^{* * *} p<0.001$. 


\subsection{IgA Is not Produced at Mucosal Sites after Vaccination with BNT162b2}

The production of $\operatorname{Ig} \mathrm{A}$ is of great interest because $\operatorname{Ig} \mathrm{A}$ is the main immunoglobulin for protection at mucosal sites, including the upper airways, which are the site of SARS-CoV-2 entry.

We measured spike-specific IgA in the serum of immunized HCWs. We found that after the first dose, $22 \%$ of the HCWs had not produced spike-specific IgA, but all of them had specific IgA at T28 in the serum. The level of IgA strongly decreased at T90 (geometric mean 12.75 at T28 and 2.6 at T90) indicating that most of the IgA plasma cells generated in response to the vaccine were short-lived (Figure 5A).

Antibodies can reach mucosal sites by transudation from the serum or can be locally produced after MBCs have migrated to mucosal sites and become plasma cells. The latter mechanism is indispensable for the secretion of IgA into breast milk [25]. Proinflammatory cytokines and chemokines are physiologically increased in the lactating breast $[26,27]$ and switched MBCs primed to secrete antibodies can be found in maternal milk. Sixteen HCWs were vaccinated after pregnancy, during lactation (Table 3). Seven days after the second vaccine dose, all of them had detectable IgA specific for spike in breast milk (Figure 5B), confirming the ability of vaccine-induced MBCs to be home to the inflammatory environment of the lactating mammary gland and locally produce IgA.

Saliva can be considered a proxy of mucosal immunity containing secretory IgA produced by local plasma cells [28]. In order to investigate whether serum IgA is associated with mucosal protection after vaccination, we measured IgA in the saliva of $43 \mathrm{HCWs}$ that had the highest IgA values in the serum at day 28.

We found that two to three weeks after the second dose, $\operatorname{IgA}$ was undetectable in the saliva of all vaccinated individuals tested (Figure 5C), demonstrating that the vaccination does not result in the presence of significant levels of IgA in the oral mucosa. Specific IgA was detectable in the saliva of $39 \mathrm{HCWs}$ previously infected with COVID-19 (Figure 5C), indicating that, as published before, the disease induces mucosal immunity [29].

Twenty-one of the 3511 vaccinated HCWs of our institute had a positive swab after completion of the two-dose vaccine cycle. All of them were related to familiar clusters. All the infected HCWs had responded to the vaccine with serum antibody production before having the positive nasopharyngeal swab. The infection was either asymptomatic or mild, without signs of pneumonia. We studied the immune response of six HCWs infected after vaccination (Table 4). Salivary IgA became detectable 0-6 days after the first positive swab and increased further after 6 days from the infection (Figure 5C). Specific IgA in the saliva was 10 times lower than IgA in the serum.

Table 4. Characteristics of HCWs infected after two doses of vaccine.

\begin{tabular}{cc}
\hline HCWs Age (Mean \pm SD) & $45.6 \pm 6.5$ \\
\hline Sex & $66.7 \% \mathrm{~F} ; 33.3 \% \mathrm{M}$ \\
\hline SARS-CoV-2 infection & $33.3 \%$ asymptomatic; $50 \%$ paucisymptomatic; $16.7 \%$ mild \\
\hline SARS-CoV-2 variant & $66.6 \%$ Gamma; $16.7 \%$ Alpha; $16.7 \%$ n.a. \\
\hline
\end{tabular}

Two of the HCWs who had a positive swab after the second vaccine dose were included in our MBC study. From the first one, we compared the samples obtained at the T28, T70, and T118 when the HCWs returned to work. During the infection, caused by a virus variant (gamma), the initial symptoms were mild, with fever, cough and muscle pain. Most of the MBCs were of low affinity (S+) at T28 and T70. S++ MBCs were increased 23 times in the last sample (Figure 5D). 


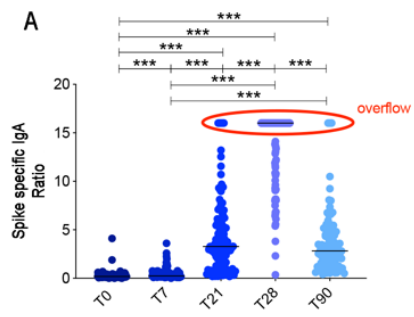

B

D

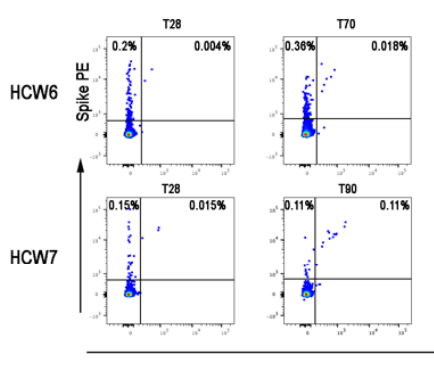

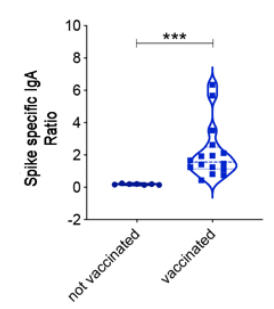
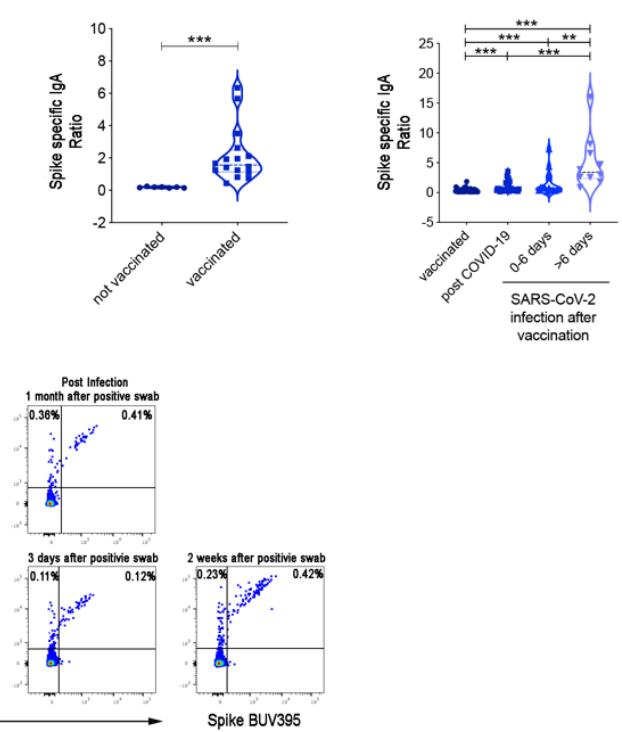

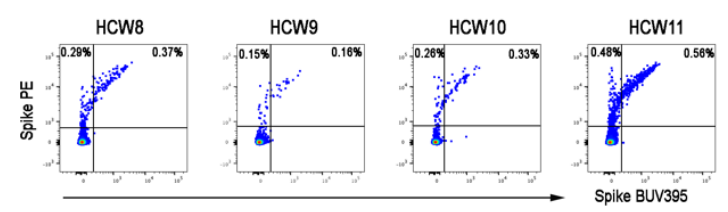

F
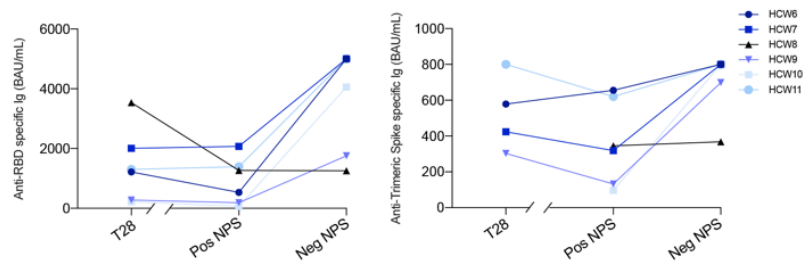

Figure 5. SARS-CoV-2 IgA antibody response. (A) Spike-specific IgA antibodies were detected by ELISA at different time points in the serum of all HCWs included in the study $(n=108)$. Overflow values are indicated by a red oval. (B) Spike-specific IgA antibodies were measured in the breast milk of 7 controls and $16 \mathrm{HCW}$ mothers vaccinated after the delivery. (C) Spike-specific IgA antibodies were measured in the saliva of 43 representative HCWs with the highest serum-specific IgA levels, in 39 HCWs who had recovered from COVID-19 and in 6 HCWs infected after two vaccine doses. In the last group, specific salivary IgA was detected at the indicated days after the first positive nasopharyngeal swab (>6 days: 7 to 15 days). (D,E) Flow cytometry plots of 6 HCWs infected after two vaccine doses showing the staining patterns of SARS-CoV-2 antigen probes on MBCs. (F) Anti-RBD and anti-trimeric $S$ titres were measured in the $6 \mathrm{HCWs}$ who had a positive nasopharyngeal swab after vaccination at T28, at the time of positive nasopharyngeal swab (Pos NPS) and when they returned to work (Neg NPS). The interrupted $x$-axis indicates that after T28, the time of the first positive NPS was variable (between day 60 and day 110 after first dose) and longer compared to the time between the positive and negative NPS (2 to 20 days). Medians are plotted, and statistical significance was determined using Wilcoxon matched-pairs signed-rank test (A) or unpaired Mann-Whitney t-test $(\mathbf{B}, \mathbf{C}){ }^{* *} p<0.01,{ }^{* * *} p<0.001$.

In contrast, the second HCW had both $\mathrm{S}+$ and $\mathrm{S}++$ MBCs at T28 and T90. A nasopharyngeal swab gave a positive result 20 days after T90, and the MBC pool remained stable in the next three days and strongly increased 2 weeks later (Figure 5D). The infection was asymptomatic in this case. 
Of four HCWs that were not included in the initial study and had a positive swab between T60 and T80, we obtained a sample when they returned to work after a paucisymptomatic disease. Their S++ were in higher frequency compared to the HCWs in the initial study group (median $=0.08 \%$, in Figure $4 \mathrm{C}$ ) suggesting that $\mathrm{MBC}$ senerated by the vaccine increase in response to the virus (Figure 5E). A further clue of the function of MBCs is the increase of serum-specific IgG after the positive swab in five out of six HCWs (Figure 5F). HCW8 was found to have a positive swab during a routine control in the absence of symptoms. The nasopharyngeal swab became negative after two days. High levels of salivary IgA (overflow) were detected at day 7 (the only sample available). Retrospectively, we found that HCW8 had antibodies against the viral nucleoprotein, already after the complete vaccine cycle, in February. We hypothesize that a previous asymptomatic and undetected infection had resulted in a powerful mucosal protection able to eliminate the virus in two days and prevent the systemic response.

\section{Discussion}

The aim of vaccination is to generate immune memory, namely the pool of cells including high-affinity MBCs and long-lived plasma cells with their antibodies that are able to prevent reinfection. Specificity and rapidity of action are the indispensable properties of protective immune memory. Differently from memory $\mathrm{T}$ cells, MBCs have the ability to improve their specificity by repeated steps of somatic mutation and selection in the GC. MBCs with high affinity for recombinant spike produce antibodies able to neutralize the virus. Monoclonal antibodies obtained after cloning from these MBCs can be used for cure and prevention of SARS-CoV-2 infection [30].

Few days after a vaccine cycle, specific antibodies peak in the serum thanks to the activity of short- and long-lived plasma cells [8]. The decay of short-lived plasma cells [8] causes the reduction of serum antibodies. As no vaccination before has been under such a constant and severe scrutiny by the public and media as this against SARS-CoV-2, the physiological drop of serum titres is seen as a sign of vaccine failure and loss of protection. In response to this anxiety, the necessity of a third vaccine dose has been suggested.

Although three months after the first dose, serum-specific antibody levels decline, we found that MBCs generated in response to vaccination continue to improve their specificity and increase in numbers (Figure 4).

By ELISpot, we show that the immune response to the vaccine generates specific MBCs expressing switched isotypes at T28, seven days after the second dose in most individuals, whereas at T21, MBCs of switched isotypes are still undetectable in $20.8 \%$ and in $41.6 \%$ of the immunized subjects for IgG and IgA, respectively (Figure 3). The abundance of IgM MBCs binding recombinant spike protein seen by ELISpot at all time-points contrasts with the appearance of IgG and IgA secreting cells only after one and two doses of vaccine. Our results can be explained considering that the ELISpot also detects low-affinity IgM and MBCs expressing and secreting this type of antibodies that plays an important role in the functional organization of the MBC pool. A large group of MBCs able to bind the antigen with low affinity is important as first step of defence. Only a few among these cells will be recruited in the GC reaction. The stringency of selection in the GC explains why very few of the IgM MBCs that bind recombinant spike in ELISpot at all time-points (median $10 \%$ ) become IgG (median $0.48 \%$ )- or IgA (median $0.09 \%$ )-secreting cells at T28. At T90, the frequency of IgG- and IgA-secreting MBCs remained stable.

In agreement with the ELISpot data, MBCs with low affinity for spike (S+) are mostly of IgM isotype and present at all time-points (Figure 4B,C), whereas high-affinity MBCs become detectable at T21 and significantly increase further at T28. They are mostly of switched isotypes (Figure 4B,C). At T90, the frequency of high-affinity MBCs is further increased, suggesting that the GC reaction continues its rounds of selection of the best specificity for months after the second vaccine dose. Low-affinity MBCs remain detectable and are still mostly of IgM isotype. MBCs able to bind RBD are about $20 \%$ of the S++ MBCs at day T28 and T90. Thus, a fraction of the response generated by immunization against 
spike protein is directed against RBD. This is important to secure the anti-viral ability of the antibodies, although the specificity of neutralizing antibodies is not exclusively limited to the RBD domain [31].

IgM MBCs with low affinity for spike (detected by FACS as S+ and also measured in ELISpot) are present at all time points. At T0, they correspond to natural/innate MBCs [10] or to MBCs involved in the response to previously encountered members of the coronavirus family [19]. IgM MBCs increasing and persisting after vaccination will be useful because they can be re-modelled in the GC in response to viral variants [11].

The first obstacle posed by the immune system to mucosal infection is secretory IgA [32]. SIgA plays a fundamental role in the protection from respiratory viruses by blocking their attachment to epithelial cells $[29,33,34]$. It has been shown that adult patients with COVID-19 have high levels of specific and neutralizing SIgA in the saliva [29]. In order to prevent viral invasion of the epithelial cells of the upper respiratory tract, we need mucosal immunity and secretory IgA [35], as demonstrated by the protective potency of maternal milk [36,37] and salivary IgA in children [38]. The absence of IgA in the saliva of vaccinated HCWs suggests that, in strict terms, the vaccine is not sterilising because it is unable to generate preventive mucosal immunity. The very low concentrations of IgA have been detected in the saliva of vaccinated individuals by using more sensitive methods that do not allow the comparison between serum and saliva IgA levels [39,40]. With our less sensitive method (the same that we have used for blood), we show the increase of salivary IgA in HCWs with a positive NPS, demonstrating the reaction to the local infection.

The lack of direct mucosal protection explains why vaccinated individuals can have a positive nasopharyngeal swab. In most cases, however, the infection remains asymptomatic or mild. Part of their defence may be due to spike-specific IgG and IgA antibodies exudated to the tissues. An important role may also be played by MBCs that migrate to the infected areas and become resident MBCs [41]. We hypothesize that specific MBCs, generated by two vaccine doses, are able to migrate to mucosal tissues and locally produce IgA antibodies, based on the observations made in two different situations, both characterised by the production of attracting inflammatory cytokines. Firstly, we show that spike-specific IgA can be detected in the breast milk of women vaccinated after pregnancy (Figure 5B). Secondly, we found that HCWs with a positive swab after the complete cycle of vaccination produce salivary IgA $2-4$ days after the first positive swab (Figure $5 \mathrm{C}$ ), suggesting that vaccine-induced $\mathrm{MBC}$ s have performed their function by rapidly migrating to the site of viral invasion. IgA in the saliva is of the secretory type, dimeric and bound to the secretory component. Dimeric IgA has a much more potent neutralization ability than serum monomeric IgA [42]. Thanks to the combined action of antibodies and MBCs (Figure 5C-E), the evolution of the disease is immediately blocked. For this reason, the viral load in vaccinated individuals who present a positive nasopharyngeal swab can be so low to be unable to transmit the disease to others [43].

The absence of cases of re-infection in the 299 HCWs who experienced COVID-19 before vaccination and have detectable levels of salivary IgA (Figure 5C) further highlights the importance of mucosal protection against SARS-CoV-2.

Mucosal vaccines against COVID-19 are in development [44] and may more effectively prevent infection and abrogate viral circulation.

The real-world effectiveness of one and two doses of RNA vaccines has just been reported by a CDC study. Among HCWs, one dose had $80 \%$ efficacy whereas two doses resulted in $90 \%$ efficacy [45]. In a different setting, in individuals over 60, after the first dose of BNT162b2 vaccine there was a significant decrease, but only of about $33 \%$ in the rate of positive tests for the coronavirus. The efficacy increased to $60 \%$ after two doses [46]. Many factors contribute to vaccine effectiveness, including age and viral circulation, but from the immunological point of view, two doses are more protective than one. The real-world effectiveness of one vaccine dose, demonstrated after day 14 post-immunization, can be explained by the performance of low-affinity MBCs and antibodies able to prevent severe 
COVID-19. Nevertheless, our data show that two doses of vaccine ensure a more protective and possibly long-lived immunological memory [47].

We show that completing the vaccination cycle is necessary to generate high levels of specific serum antibodies and MBCs. A single dose of vaccine may result in an imperfect immunological memory lacking the strength (high-affinity MBCs and antibodies) to rapidly and completely clear the virus. The persistence of replicating viruses in a partially protected patient might lead to the evolution and selection of viral variants. Variants rapidly spread and weaken the protective potentials of the vaccines that we already have and of those that are still in development.

The robustness of vaccine-induced protection does not depend only on the amount of serum antibodies but mostly on the persistence of MBCs able to migrate to the site of infection, locally produce antibodies and remodel in response to viral variants. IgM MBCs that have acquired the specificity for spike protein may be the substrate for further modifications and adaptation to viral variants, thus ensuring a rapid response. In agreement with our hypothesis, vaccinated HCWs who had a positive nasopharyngeal swab experienced a pauci or asymptomatic infection by gamma or alpha VoC. Further studies are necessary to investigate the duration and resilience of the immune memory, both B- and T-cell memory, induced by the available vaccines.

For all these reasons, we should not worry about the antibody decline in the serum, observed a few months after vaccination or natural infection. Our MBCs, along with memory $\mathrm{T}$ cells, are an essential defence weapon that ensure local and systemic protection after re-encounter with the antigen. In conclusion, as with any other vaccine produced before, RNA-vaccines appear to be able to generate a typical immunological memory that is predictably stable and durable.

Supplementary Materials: The following are available online at https:/ /www.mdpi.com/article/10 .3390/cells10102541/s1, Figure S1: Spike-specific memory B cells in convalescent COVID-19 patients, Table S1: Antibodies for staining.

Author Contributions: Conceptualization: R.C., E.P.M., C.R., M.R.V., S.T., A.F.S., C.F.P., S.Z., T.C.; Methodology: E.P.M., S.T., A.F.S., C.A. (Christian Albano), P.R., S.M., G.R., E.P., C.R., L.P., L.C. (Luna Colagrossi), C.C., S.R., S.M., M.R.V., R.B., D.G., V.C., A.S., M.L.C.D.A., N.R., G.S., N.C., D.A., C.A. (Claudia Alteri), L.C. (Luana Coltella), A.R., N.C., D.A., M.A.; Data interpretation: R.C., E.P.M., S.T., A.F.S., G.L., C.R., M.R.V.; Funding acquisition: R.C., C.A. (Chiara Agrati), F.L., C.F.P., S.Z.; Project administration: R.C., C.F.P., S.Z.; Supervision: R.C., C.F.P., S.Z.; Writing一original draft: R.C., E.P.M., S.T., A.F.S.; Writing-review and editing: R.C., E.P.M., S.T., A.F.S., F.L., C.F.P., S.Z., N.M., M.R.V., C.R. All authors have read and agreed to the published version of the manuscript.

Funding: This work was funded by: Italian Ministry of Health RF2013-02358960 grant, Italian Ministry of Health COVID-2020-12371817 grant, Ricerca Corrente 2021 “5 per mille".

Institutional Review Board Statement: Not applicable.

Informed Consent Statement: Not applicable.

Data and Materials Availability: All data are available in the main text or the Supplementary Materials.

Acknowledgments: We acknowledge the nurse staff for their support of the vaccination campaign and all the HCWs that have been enrolled for the study.

Conflicts of Interest: Authors declare that they have no competing interests.

\section{References}

1. Polack, F.P.; Thomas, S.J.; Kitchin, N.; Absalon, J.; Gurtman, A.; Lockhart, S.; Perez, J.L.; Pérez Marc, G.; Moreira, E.D.; Zerbini, C.; et al. Safety and Efficacy of the BNT162b2 MRNA Covid-19 Vaccine. N. Engl. J. Med. 2020, 383, 2603-2615. [CrossRef]

2. Jackson, L.A.; Anderson, E.J.; Rouphael, N.G.; Roberts, P.C.; Makhene, M.; Coler, R.N.; McCullough, M.P.; Chappell, J.D.; Denison, M.R.; Stevens, L.J.; et al. An MRNA Vaccine against SARS-CoV-2-Preliminary Report. N. Engl. J. Med. 2020, 383, $1920-1931$. [CrossRef] 
3. Voysey, M.; Clemens, S.A.C.; Madhi, S.A.; Weckx, L.Y.; Folegatti, P.M.; Aley, P.K.; Angus, B.; Baillie, V.L.; Barnabas, S.L.; Bhorat, Q.E.; et al. Safety and Efficacy of the ChAdOx1 NCoV-19 Vaccine (AZD1222) against SARS-CoV-2: An Interim Analysis of Four Randomised Controlled Trials in Brazil, South Africa, and the UK. Lancet 2021, 397, 99-111. [CrossRef]

4. Mathieu, E.; Ritchie, H.; Ortiz-Ospina, E.; Roser, M.; Hasell, J.; Appel, C.; Giattino, C.; Rodés-Guirao, L. A Global Database of COVID-19 Vaccinations. Nat. Hum. Behav. 2021, 5, 947-953. [CrossRef]

5. Bieniasz, P. The Case against Delaying SARS-CoV-2 MRNA Vaccine Boosting Doses. Clin. Infect. Dis. Off. Publ. Infect. Dis. Soc. Am. 2021. Online ahead of print. [CrossRef]

6. Saad-Roy, C.M.; Morris, S.E.; Metcalf, C.J.E.; Mina, M.J.; Baker, R.E.; Farrar, J.; Holmes, E.C.; Pybus, O.G.; Graham, A.L.; Levin, S.A.; et al. Epidemiological and Evolutionary Considerations of SARS-CoV-2 Vaccine Dosing Regimes. Science 2021, 372, 363-370. [CrossRef]

7. Andreano, E.; Rappuoli, R. SARS-CoV-2 Escaped Natural Immunity, Raising Questions about Vaccines and Therapies. Nat. Med. 2021, 27, 759-761. [CrossRef]

8. Pollard, A.J.; Bijker, E.M. A Guide to Vaccinology: From Basic Principles to New Developments. Nat. Rev. Immunol. 2021, 21, 83-100. [CrossRef]

9. Yoshida, T.; Mei, H.; Dörner, T.; Hiepe, F.; Radbruch, A.; Fillatreau, S.; Hoyer, B.F. Memory B and Memory Plasma Cells. Immunol. Rev. 2010, 237, 117-139. [CrossRef]

10. Aranburu, A.; Piano Mortari, E.; Baban, A.; Giorda, E.; Cascioli, S.; Marcellini, V.; Scarsella, M.; Ceccarelli, S.; Corbelli, S.; Cantarutti, N.; et al. Human B-Cell Memory Is Shaped by Age- and Tissue-Specific T-Independent and GC-Dependent Events. Eur J. Immunol. 2017, 47, 327-344. [CrossRef]

11. Grimsholm, O.; Piano Mortari, E.; Davydov, A.N.; Shugay, M.; Obraztsova, A.S.; Bocci, C.; Marasco, E.; Marcellini, V.; Aranburu, A.; Farroni, C.; et al. The Interplay between CD27dull and CD27bright B Cells Ensures the Flexibility, Stability, and Resilience of Human B Cell Memory. Cell Rep. 2020, 30, 2963-2977. [CrossRef]

12. Crotty, S. A Brief History of T Cell Help to B Cells. Nat. Rev. Immunol. 2015, 15, 185-189. [CrossRef]

13. Relative Fluorochrome Brightness. Available online: https://www.bu.edu/flow-cytometry / files/2014/09/Fluorochrome-ChartRelative-Brightness.pdf (accessed on 17 May 2021).

14. Marasco, E.; Farroni, C.; Cascioli, S.; Marcellini, V.; Scarsella, M.; Giorda, E.; Piano Mortari, E.; Leonardi, L.; Scarselli, A.; Valentini, D.; et al. B-Cell Activation with CD40L or CpG Measures the Function of B-Cell Subsets and Identifies Specific Defects in Immunodeficient Patients. Eur. J. Immunol. 2017, 47, 131-143. [CrossRef]

15. Marcellini, V.; Piano Mortari, E.; Fedele, G.; Gesualdo, F.; Pandolfi, E.; Midulla, F.; Leone, P.; Stefanelli, P.; Tozzi, A.E.; Carsetti, R.; et al. Protection against Pertussis in Humans Correlates to Elevated Serum Antibodies and Memory B Cells. Front. Immunol. 2017, 8, 1158. [CrossRef]

16. Carsetti, R.; Zaffina, S.; Piano Mortari, E.; Terreri, S.; Corrente, F.; Capponi, C.; Palomba, P.; Mirabella, M.; Cascioli, S.; Palange, P.; et al. Different Innate and Adaptive Immune Responses to SARS-CoV-2 Infection of Asymptomatic, Mild, and Severe Cases. Front. Immunol. 2020, 11, 610300. [CrossRef]

17. Dan, J.M.; Mateus, J.; Kato, Y.; Hastie, K.M.; Yu, E.D.; Faliti, C.E.; Grifoni, A.; Ramirez, S.I.; Haupt, S.; Frazier, A.; et al. Immunological Memory to SARS-CoV-2 Assessed for up to 8 Months after Infection. Science 2021, 371, eabf4063. [CrossRef]

18. Ochsenbein, A.F.; Zinkernagel, R.M. Natural Antibodies and Complement Link Innate and Acquired Immunity. Immunology Today 2000, 21, 624-630. [CrossRef]

19. Selva, K.J.; van de Sandt, C.E.; Lemke, M.M.; Lee, C.Y.; Shoffner, S.K.; Chua, B.Y.; Davis, S.K.; Nguyen, T.H.O.; Rowntree, L.C.; Hensen, L.; et al. Systems Serology Detects Functionally Distinct Coronavirus Antibody Features in Children and Elderly. Nat. Commun. 2021, 12, 2037. [CrossRef]

20. Throsby, M.; van den Brink, E.; Jongeneelen, M.; Poon, L.L.M.; Alard, P.; Cornelissen, L.; Bakker, A.; Cox, F.; van Deventer, E.; Guan, Y.; et al. Heterosubtypic Neutralizing Monoclonal Antibodies Cross-Protective against H5N1 and H1N1 Recovered from Human IgM ${ }^{+}$Memory B Cells. PLoS ONE 2008, 3, e3942. [CrossRef]

21. Agrati, C.; Castilletti, C.; Goletti, D.; Meschi, S.; Sacchi, A.; Matusali, G.; Bordoni, V.; Petrone, L.; Lapa, D.; Notari, S.; et al. Coordinate Induction of Humoral and Spike Specific T-cell Response in a Cohort of Italian Health Care Workers Receiving Bnt162b2 Mrna Vaccine. Microorganisms 2021, 9, 1315. [CrossRef]

22. Kohmer, N.; Rühl, C.; Ciesek, S.; Rabenau, H.F. Utility of Different Surrogate Enzyme-Linked Immunosorbent Assays (SELISAs) for Detection of SARS-CoV-2 Neutralizing Antibodies. J. Clin. Med. 2021, 10, 2128. [CrossRef]

23. Siegrist, C.-A. Vaccine Immunology. In Plotkin's Vaccine, 7th ed.; Plotkin, S.A., Orenstein, W.A., Offit, P.A., Edwards, K., Eds.; Elsevier: Amsterdam, Netherlands, 2018; pp. 16-34.

24. Victora, G.D.; Wilson, P.C. Germinal Center Selection and the Antibody Response to Influenza. Cell 2015, 163, 545-548. [CrossRef]

25. Goldman, A.S.; Chheda, S.; Keeney, S.E.; Schmalstieg, F.C. Immunology of Human Milk and Host Immunity. Fetal Neonatal Physiol. 2011, 1690-1701. [CrossRef]

26. Ferrari, D.V.D.J.; Polettini, J.; de Moraes, L.L.; de Campos, L.A.; da Silva, M.G.; Saeki, E.K.; Morceli, G. Profile of Pro-Inflammatory Cytokines in Colostrum of Nursing Mothers at the Extremes of Reproductive Age. PLoS ONE 2020, 15, e0231882. [CrossRef]

27. Michie, C.A.; Tantscher, E.; Schall, T.; Rot, A. Physiological Secretion of Chemokines in Human Breast Milk. Eur. Cytokine Netw. 1998, 9, 123-129.

28. Brandtzaeg, P. Secretory Immunity with Special Reference to the Oral Cavity. J. Oral Microbiol. 2013, 5. [CrossRef] 
29. Sterlin, D.; Mathian, A.; Miyara, M.; Mohr, A.; Anna, F.; Claër, L.; Quentric, P.; Fadlallah, J.; Devilliers, H.; Ghillani, P.; et al. IgA Dominates the Early Neutralizing Antibody Response to SARS-CoV-2. Sci. Transl. Med. 2020, 13, eabd2223. [CrossRef]

30. Andreano, E.; Nicastri, E.; Paciello, I.; Pileri, P.; Manganaro, N.; Piccini, G.; Manenti, A.; Pantano, E.; Kabanova, A.; Troisi, M.; et al. Extremely Potent Human Monoclonal Antibodies from COVID-19 Convalescent Patients. Cell 2021, 184, 1821-1835.e16. [CrossRef]

31. Barnes, C.O.; Jette, C.A.; Abernathy, M.E.; Dam, K.-M.A.; Esswein, S.R.; Gristick, H.B.; Malyutin, A.G.; Sharaf, N.G.; Huey-Tubman, K.E.; Lee, Y.E.; et al. SARS-CoV-2 Neutralizing Antibody Structures Inform Therapeutic Strategies. Nature 2020, 588, 682-687. [CrossRef]

32. Kumar, N.; Arthur, C.P.; Ciferri, C.; Matsumoto, M.L. Structure of the Secretory Immunoglobulin A Core. Science 2020, 367, eaaz5807. [CrossRef]

33. Mazanec, M.B.; Coudret, C.L.; Fletcher, D.R. Intracellular Neutralization of Influenza Virus by Immunoglobulin A AntiHemagglutinin Monoclonal Antibodies. J. Virol. 1995, 69, 1339-1343. [CrossRef] [PubMed]

34. Devito, C.; Hinkula, J.; Kaul, R.; Lopalco, L.; Bwayo, J.J.; Plummer, F.; Clerici, M.; Broliden, K. Mucosal and Plasma IgA from HIV-Exposed Seronegative Individuals Neutralize a Primary HIV-1 Isolate. AIDS (Lond. Engl.) 2000, 14, 1917-1920. [CrossRef] [PubMed]

35. Quinti, I.; Mortari, E.P.; Fernandez Salinas, A.; Milito, C.; Carsetti, R. IgA Antibodies and IgA Deficiency in SARS-CoV-2 Infection. Front. Cell. Infect. Microbiol. 2021, 11, 257. [CrossRef]

36. Tuaillon, E.; Valea, D.; Becquart, P.; Al Tabaa, Y.; Meda, N.; Bollore, K.; Van de Perre, P.; Vendrell, J.-P. Human Milk-Derived B Cells: A Highly Activated Switched Memory Cell Population Primed to Secrete Antibodies. J. Immunol. 2009, 182, 7155-7162. [CrossRef]

37. Fox, A.; Marino, J.; Amanat, F.; Krammer, F.; Hahn-Holbrook, J.; Zolla-Pazner, S.; Powell, R.L. Robust and Specific Secretory IgA Against SARS-CoV-2 Detected in Human Milk. iScience 2020, 23, 101735. [CrossRef]

38. Tosif, S.; Neeland, M.R.; Sutton, P.; Licciardi, P.V.; Sarkar, S.; Selva, K.J.; Do, L.A.H.; Donato, C.; Quan Toh, Z.; Higgins, R.; et al. Immune Responses to SARS-CoV-2 in Three Children of Parents with Symptomatic COVID-19. Nat. Commun. 2020, 11, 5703. [CrossRef]

39. Ketas, T.J.; Chaturbhuj, D.; Portillo, V.M.C.; Francomano, E.; Golden, E.; Chandrasekhar, S.; Debnath, G.; Díaz-Tapia, R.; Yasmeen, A.; Kramer, K.D.; et al. Antibody Responses to SARS-CoV-2 MRNA Vaccines Are Detectable in Saliva. Pathog. Immun. 2021, 6, 116-134. [CrossRef]

40. Becker, M.; Dulovic, A.; Junker, D.; Ruetalo, N.; Kaiser, P.D.; Pinilla, Y.T.; Heinzel, C.; Haering, J.; Traenkle, B.; Wagner, T.R.; et al. Immune Response to SARS-CoV-2 Variants of Concern in Vaccinated Individuals. Nat. Commun. 2021, 12, 3109. [CrossRef]

41. Allie, S.R.; Bradley, J.E.; Mudunuru, U.; Schultz, M.D.; Graf, B.A.; Lund, F.E.; Randall, T.D. The Establishment of Resident Memory B Cells in the Lung Requires Local Antigen Encounter. Nat. Immunol. 2019, 20, 97-108. [CrossRef]

42. Wang, Z.; Lorenzi, J.C.C.; Muecksch, F.; Finkin, S.; Viant, C.; Gaebler, C.; Cipolla, M.; Hoffmann, H.-H.; Oliveira, T.Y.; Oren, D.A.; et al. Enhanced SARS-CoV-2 Neutralization by Dimeric IgA. Sci. Transl. Med. 2020, 13, eabf1555. [CrossRef]

43. Levine-Tiefenbrun, M.; Yelin, I.; Katz, R.; Herzel, E.; Golan, Z.; Schreiber, L.; Wolf, T.; Nadler, V.; Ben-Tov, A.; Kuint, J.; et al. Initial Report of Decreased SARS-CoV-2 Viral Load after Inoculation with the BNT162b2 Vaccine. Nat. Med. 2021, $27,790-792$. [CrossRef] [PubMed]

44. Mudgal, R.; Nehul, S.; Tomar, S. Prospects for Mucosal Vaccine: Shutting the Door on SARS-CoV-2. Hum. Vaccines Immunother. 2020, 16, 2921-2931. [CrossRef] [PubMed]

45. Thompson, M.G.; Burgess, J.L.; Naleway, A.L.; Tyner, H.L.; Yoon, S.K.; Meece, J.; Olsho, L.E.W.; Caban-Martinez, A.J.; Fowlkes, A.; Lutrick, K.; et al. Interim Estimates of Vaccine Effectiveness of BNT162b2 and MRNA-1273 COVID-19 Vaccines in Preventing SARS-CoV-2 Infection Among Health Care Personnel, First Responders, and Other Essential and Frontline Workers-Eight U.S. Locations, December 2020-March. MMWR. Morb. Mortal. Wkly. Rep. 2021, 70, 495-500. [CrossRef] [PubMed]

46. Mahase, E. Covid-19: Reports from Israel Suggest One Dose of Pfizer Vaccine Could Be Less Effective than Expected. BMJ 2021, 372, n217. [CrossRef]

47. Takemori, T. B cell Memory and Plasma Cell Development. In Molecular Biology of B Cells, 2nd ed.; Honjo, T., Radbruch, A., Reth, M., Alt, F., Eds.; Elsevier: London, UK, 2015; pp. 227-249. 